\title{
Review of the Combination Strategies Used in Anti-PD1/PD-L1 Monoclonal Antibody Treatment
}

\author{
Jiaxing Cheng* \\ University of Nottingham, Medical School, Nottingham, NG7 2UH, UK
}

\begin{abstract}
In recent years, the research achievements of immunotherapy were outstanding, especially in the field of cancer treatment. At present, the major breakthroughs have been made in the research of immunotherapy based on programmed death receptor 1 (programmed death 1, PD-1) or programmed death ligand 1 (programmed death-ligand 1, PD-L1) immune checkpoint blockage. The successful application of anti-PD-1/PD-L1 mono clonal antibody in various clinical trials has showed its remarkable potential in caner immunotherapy. However, the results were not always satisfactory that treatment results often show large individual differences between patients, and it's usually only a small part of patients would respond the treatment. Thus, combination therapy has become a new research focus in anti-PD-1/PD-L1 blockagebased therapy strategy development. This article will review the combination strategies of anti-PD-1/PD-L1 mAbs treatment and discuss the development and the trend of combination immunotherapy.
\end{abstract}

\section{Introduction}

In the last decade, immunotherapy was developed rapidly, especially in the field of cancer treatment. It became an effective strategy to treat cancer, even has been regarded as the hope of human to defeat cancer. The aim of cancer immunotherapy is to induce a durable immune response, which may kill the tumor cells and suppress the cancer progression in the long term. But immunotherapy is not always invincible, the main limitation of cancer immunotherapy comes from the immune suppression and tumor immune escape. The programmed death-1 (PD-1) and programmed deathligand 1 (PD-L1) play a vital role in immune suppression and the process of tumor escape. PD-1 is a critical member of the immune regulatory molecular family, usually expressed on the surface T-cells and works as a co-receptor [1]. PD-L1 usually expressed on healthy cells and prevented them from Immunol attacking. Once PD-1 and PD-L1 bind together, an inhibitory signal will be induced into T-cell to stop the intracellular immune activities and inhibit the T-cell mediated immune response [2]. In a healthy condition, this immune regulatory activity helps to protect normal tissue cells from the damage caused by the uncontrolled immune response. When the tumor cells express PD-L1, this immune regulatory activity will protect the tumor cells, and they can escape from immune surveillance. Thus, disrupt the engagement of PD-1 and PD-L1 is essential to activate the T-cell function, boost the immune response against tumor cells and keep the long-term tumor specific immune response [3].

PD-1 had been first found in 1992, and the engagement of PD-1 and PD-L1 was identified in 2000.
In 2007, the first anti-PD-1 mAb reached to clinical trials and 2 anti-PD-1 monoclonal antibodies (mAbs), pembrolizumab and nivolumab, has been approved for melanoma treatment in 2014 [4]. Now, 9 types of antiPD-1 or PD-L1 mAbs have been approved and mainly been used to treat 16 different types of cancer, including, NSCLC, Hodgkin's lymphoma, melanoma, Renal cell carcinoma and bladder cancer, and tissue-agnostic indication (Table.1). During the treatment, anti-PD1/PD-L1 mAb shows a remarkable ability to shrink tumors and induce a persistent tumor-specific immune response and tolerability in various types of cancer. However, they are not valid for all types of cancers, and still only a relatively small percentage of patients respond to this therapy strategy, some patients might be suffering from tumor resistance to PD-1/PD-L1 mAb treatment after the first response [5]. Thus, combination therapy becomes a new trend in the field of PD-1/PD-L1 $\mathrm{mAb}$ research.

Nowadays, thousands of clinical trials were performed to testing and evaluating the clinical activity of the PD-1/PD-L1 mAbs treatment as monotherapies or combined with other cancer therapy strategies [6]. The most popular combination is combining anti-PD-1/PDL1 mAb with chemotherapy. Another popular way is combining with another checkpoint inhibitor, such as CTLA-4 inhibition [7]. The outcomes of combination therapy in these researches were different based on the way of combination and cancer stage or types, and some research gets a very satisfactory result. Thus, combination therapy gives a new idea to improve antiPD-1/PD-L1 mAb therapy. This article will review the clinical efficacy of PD-1 inhibitors at immune checkpoints as well as their influencing factors,

\footnotetext{
*Jiaxing Cheng: 1941285389@qq.com
} 
mechanism of action, and research status of PD-1 inhibitors in combination with other treatments.

Table 1. The approved anti-PD1 or PD-L1 mAbs in the world wide. Four new PD-1 monoclonal antibodies (mAbs) entered the market last year, bringing the total number of listings on the global market to nine.

\begin{tabular}{|c|c|c|c|}
\hline Name of mAb & Company & Target & Time to market \\
\hline Nivolumab & BMS & PD-1 & 2014 \\
\hline Pembrolizumab & Merck & PD-1 & 2014 \\
\hline Atezolizumab & Roche & PD-L1 & 2016 \\
\hline Avelumab & Pfizer/Merck KGaA & PD-L1 & 2017 \\
\hline Durvalumab & AstraZeneca & PD-L1 & 2017 \\
\hline Cemiplimab & Jiangsu Henghui Medicine & PD-1 & 2019 \\
\hline Camrelizumab & Regeneron & PD-1 & 2019 \\
\hline Sintilimab & Innovent Biologics/Eli Lilly & PD-1 & 2019 \\
\hline Toripalimab & Junshi Biosciences & PD-1 & 2019 \\
\hline
\end{tabular}

\section{Anti-PD1/PD-L1 mAb with traditional strategy (chemotherapy, radio and chemo-radiotherapy)}

Chemotherapy and radiotherapy have been used as the standard first-line therapy for varied types of cancer and shown the remarkable successes in killing cancer cells, shrinking the tumor and inhibiting metastasis. Despite the achievements in cancer treatment, their side effects are non-negligible. Chemotherapy killing and control the metastasis of tumor cells is based on the cytotoxic drugs, the main side effects come from the cytotoxicity of these drugs to the normal healthy cells. The most side effects are affecting the gastrointestinal function and hematopoietic function, patients may suffer from nausea, vomiting, hair loss and decreased white blood cells and platelets [8]. Radiotherapy also is known as electrotherapy that use the high-energy radiation to kill the tumor cells. The local skin and mucous membrane may be affected and damaged, and the treatment region also limited within the irradiation region. Another disadvantage of both chemo and radiotherapy is the poor prognosis and challenging to build a long-term immune response. Patients have a high probability of getting recurrence after the first treatment [9].

Some studies showed that the expression of PD-L1 on tumor cells was up-regulated after chemotherapy, and the abscopal effect of radiotherapy also indicates the ability to stimulate the tumor specific immune response. These findings are the potential opportunities to combine chemo or radiotherapy with the immunotherapy. According to the analysis of combination trials in the past two years, combined chemotherapy and radiotherapy with PD1/PD-L1 inhibitor became the hot spot on new combination therapy strategy researches.

The results of chemotherapy researches suggest that induce a tumor specific response by killing the immunogenic cells and destroy the immunosuppressive microenvironment [10]. In the study of colon cancer, during the immunogenic cell death in oxaliplatin treatment, calreticulin explored on the tumor cell surface, high mobility group box protein 1 (HMGB1) and ATP released from the dead tumor cells, which can stimulate the maturation and activation of dendritic cells (DCs)and an antitumor immune response has been induced [11]. In preclinical studies, there is increasing evidence suggests that chemotherapy can stimulate the immune system activating, cause an antitumor immune response and improving the efficacy of these drugs [12], and combining PD-1 or PD-L1 mAbs with chemotherapy have shown the treatment induced an excellent antitumor activity to against advanced NSCLC in early clinical trials $[13,14]$. Also, chemotherapy with platinum can promote the maturation and activation of APCs, increase the level of tumor infiltrating $\mathrm{T}$ cells at the tumor site, and downregulate the level of MDSCs and Tregs $[15,16]$, which will make the tumor sensitive to PD-1/PD-L1 blockade.

Based on the findings of these researches, various clinical trials have been designed and performed to testing and evaluating the efficacy and o safety of the combination combined platinum-based chemotherapy with PD1/PD-L1 mAbs. A study on NSCLC treatment (phase I/II trial) combined pembrolizumab, carboplatin, paclitaxel, pemetrexed and bevacizumab in different combinations. The results demonstrated that different combination has different ORR with the median followup period of 12 months and pembrolizumab with carboplatin, and pemetrexed is the most effective combination that the ORR was about $70 \%$ [17]. Similar to the clinical trial of pembrolizumab, a phase I trial of combination therapy with nivolumab also performed among patients with NSCLC. The data shows that the frequency of progressive disease is lower, and the ORR is higher, and patients with PD-L1 level that PD-L1 $\geq 1 \%$ show that the combination of nivolumab and chemotherapy can improve the prognosis and prolong the survival of patients with advanced NSCLC [17]. All these results make people believe that the combination of chemotherapy and immunotherapy is the next step in cancer treatment.

Similar to chemotherapy, radiotherapy is using radiation to destroy the tumor cells in a loco region, and it also became a prevalent role in combination therapy researches in these years. Despite the of its limitation of effect region, many studies have shown that radiotherapy can induce the antitumor response because the dead cells will release intercellular contents like ATP and other proteins, which can be recognized as a danger signal and cause the activation of APCs. The tumor specific antigens also will be released during cell death [18]. Once the tumor specific antigen has been taking by APCs, president to $\mathrm{T}$ cells and activate them, the antitumor immunity will be generated. And these findings could be the foundation to combine radiotherapy with anti-PD1/PD-L1 mAbs.

In vitro studies of combination therapy, the results suggest that when using radiation and anti-PD-L1 mAbs together, the antitumor activities have been boosted $[19,20]$. Another study in a mouse model found that radiation therapy (RT) can upregulate the expression of 
PD-L1 on DCs and enhance the antigen crosspresentation in lymph nodes, which activity may promote the antitumor immune response [21,22]. Preclinical research also indicates that irradiated effector $T$ cells can induce a reduction in the number of tumor cells expressing PD-L1, which suggests that the combination of RT and anti-PD-L1 mAb can enhance the antitumor effect of RT [23]. Furthermore, a clinical trial indicated that patients with metastatic brain melanoma had an increased 1-year overall survival (OS) when they got nivolumab treatment during or after RT [24].

\section{PD1/PD-L1 mAb with other mAbs}

\subsection{CTLA-4}

The cytotoxic T-lymphocytes-associated antigen 4 (CTLA-4) is another important target in immune checkpoint inhibition therapy. Similar to PD-1, it plays a crucial role in the negative regulation of T-cell functions. The difference between them is the inhibition effect on T-cells, and the negative signal from CTLA-4 signaling doesn't kill the T-cell, it usually suppresses the cell's function only. CTLA-4 is a homolog of CD28 that has a much higher binding affinity with B7 [25]. The competitive interaction of CTLA-4 and B7 will prevent the co-stimulatory signal from CD28/B7 binding [26]. The relative proportion of CTLA-4/B7 and CD28/B7 binding determines whether a $\mathrm{T}$ cell will be activated or not. Another aspect of CTLA-4 immune suppression function is Tregs constitutively express CTLA-4, and the binding of CTLA-4 and B7 is essential for their suppressive functions, which is an opposite situation of T-cell activation and proliferation [27]. Due to all these reasons, anti-CTLA-4 mAbs became an important role in cancer immune therapy due to their ability to maintain the activation and proliferation of effector $\mathrm{T}$ cell, also inhibits the immune suppression effects from Tregs.

When using anti-CTLA-4 and PD-1 mAbs in the monotherapy way, the results have shown an increased survival time of responding to patients significantly. However, the benefits of them were limited by the low response rate, for example, more than $50 \%$ metastasis melanoma patients do not respond to the treatment that the objective response rate (ORR) for ipilimumab is 10 $16 \%$ and for nivolumab and pembrolizumab is $30-40 \%$ $[14,28,29]$. Therefore, it is recommended to use antiCTLA-4 mAb and anti-PD-1 mAb in combination to improve the patient's remission rate and survival rate. It is believed that CTLA-4 blockade, which is mainly involved in regulating $\mathrm{T}$ cell activation in lymph nodes or tissues and inhibits DC activity by Treg cells, has a synergistic effect with PD-1, which is primarily involved in PD-1 blockade. Inhibits the activation of effector $T$ cells and NK cells in peripheral tissues and induces Treg cell differentiation [30-32]

Combine CTLA-4 and PD-1 became a hot topic in the field of combination therapy researches, about 200 new trials have been started or done in the past 2 years. This combination strategy keeps the upstream process (antigen-presenting and $\mathrm{T}$ cell activation) and the downstream process (effector $\mathrm{T}$ cells to overcome the immunosuppressive environment around the tumor) of the whole antitumor immune response. The most results of these studies also suggest that this combination works better than monotherapy in different cancer like NSCC, metastasis melanoma and colorectal cancer.

In the field of melanoma treatment, in a phase 1 study, ipilimumab and nivolumab have been combined and been tested and evaluated. The results show that the ORR increased to $61 \%$, with $22 \%$ of patients have complete response27. Another phase II study also demonstrated that the 2-years overall survival (OS) rate increased to $63.8 \%$ at the median of follow-up period when the patients get the combinational treatment with anti-PD-1 and CTLA-4 mAbs [33]. Results from another study of advanced melanoma, which analyzed the results after 3 and 4-year follow-up of the patients, showed the superior benefits of combination therapy over monotherapy $[34,35]$.

The combination of nivolumab and ipilimumab also has been evaluated in the trial of metastatic renal cell carcinoma. The phase I study tested different multipledose groups of the combination, the result there was no statistically significant difference between different clusters [36]. Then, the outcome of the randomized phase III study suggested that patients treated with combination therapy have increased 18 months OS rate as $75 \%$ and ORR of $42 \%$. In the follow-up, the analysis showed that patients get combination therapy in phase III trial had had the better health-related quality of and life fewer symptoms compared to the control group [35,37].

\subsection{4-1BB}

4-1BB also called as CD137, which is a member of TNFR superfamily 9, mainly expressed on activated T cells or NK cells and plays a role as an inducible costimulatory receptor [38]. 4-1BBL is the ligand of 4-1BB, usually expressed on the surface of activated APCs, the binding of 4-1BB and 4-1BBL will promote cell survival, cytokine production and proliferation of $\mathrm{T}$ cells and enhance the effector function. The activation of NK cells will induce a stronger antibody-dependent cell-mediated toxicity $[39,40]$. Furthermore, the previous study investigated that the dual immunomodulatory activity of 4-1BB provides the possibility of enhancing antitumor activity while suppressing autoimmune side effects associated with immunotherapy that disrupts immune tolerance [41]. Thus, the agonistic 4-1BB mAbs has the potential to be a perfect agent to be used in combinational therapy with other therapeutic mAbs. The research with a poorly immunogenic melanoma mouse model has investigated that anti-4-1BB/anti-PD-1 combination stimulated the differentiation of effector and memory T-cell, promoted the antitumor activities in the tumor microenvironment and generated an antigenspecific CTL response [42]. All the results denoted the remarkable potential of anti-4-1BB/anti-PD-1 combination in cancer treatment. 
Currently, two agonists 4-1BB mAb have been approved in clinical studies, including Bristol-Myers Squibb's urelumab and Pfizer's utomilumab. The use of these antibodies alone or in combination with other tumor treatment methods can significantly improve the efficiency of cancer treatment. Utomilumab is a fully human monoclonal antibody that can stimulate 4-1BB. In phase I clinical trials, it has been used in combination with pembrolizumab among patients with advanced solid tumors. No dose-limiting toxicity has been reported, and about a quarter of patients have different levels of response. Urelumab is another 4-1BB agonist antibody that has been evaluated in clinical trials in patients with various advanced solid tumors. The results of safety analysis of these trials demonstrated that when using large doses of the drug, the drug sometimes caused severe ammonia. Currently, the combination of this drug with nivolumab has been evaluated in the patients with B-cell non-Hodgkin lymphoma and solid tumors in phase I and II clinical trials. Preliminary results show that $6 / 60$ of patients with lymphoma treated with urine monoclonal antibodies achieved partial response (PR) or complete response (CR), and 9 of 86 patients receiving combination therapy achieved PR. However, patients without NSCLC or NSCLC reported a diffuse large Bcell lymphoma reaction. It is worth noting that at least $3 \%$ of patients have grade 3 to 4 ammonia, while about $7 \%$ of the 123 patients suffering from serious adverse events, and $5 \%$ of study patients discontinuing the drug due to this reason [43].

\section{$3.3 \mathrm{CD} 38$}

CD 38 is a kind of ectoenzyme that mainly expressed on the surface of tumor cells and activated immune cells such as B cells, T cells, NK cells, MDSC and Tregs. CD38 will promote adenosine generation through the CD38-CD203a-CD73 pathway [44], then, adenosine will bind to the adenosine receptors (A2AR and A2BR) on $\mathrm{T}$ cells and induce the suppression of immune activity and led to the resistance of PD-1/PD-L1 mAb treatment. Thus, CD38 blockades could be a strategy that helps patients to overcome the Acquired PD-1/PD-L1 resistance. Chen's team used a lung cancer mouse model, compared the different combinations of PD-L1 with CD38 or PD-L1 with A2AR/A2BR antagonists, confirmed that the combination of anti-PD-L1 and anti CD38 antibodies has a remarkable therapeutic benefit on inhibition of tumor cell growth and metastasis of primary lung cancer [45]. Another study with multiple myeloma (MM) mouse model also proved this combination was more effective than the monotherapy of any one of these mAbs [46]. Thus, based on these results of preclinical researches, this combination could be an excellent strategy to use in cancer treatment.

\subsection{Bispecific antibody}

In addition to the strategy of directly binding anti-PD1 / PD-L1 mAb to other antibodies, the production of bispecific antibodies is a new method of combining two different antibodies. Compared with two separate mAb combinations, bispecific antibodies (BsAb) as a method of simultaneously targeting two different antigens is an emerging method to improve clinical efficacy. In immuno-oncology, BsAbs can increase the antitumor efficiency and avoid drug resistance compared with the mAbs combinations, because through the affinity effect and the selective activation of the immune system, the two immune modulation pathways can antagonize the system more within the tumor microenvironment.

William's team developed a novel BsAb that targeting both RANKL and PD-1. RANK (TNFRSF11a) and RANKL (TNFSF11) are members of the tumor necrosis factor receptor and ligand superfamilies, respectively, with the closest homology to CD40 and CD40L [47]. RANK/RANKL interaction has been proved that tumor progression and immunotherapy resistance [48]. William and his collagens used a mouse model to test this $\mathrm{BsAb}$, and the results showed that the antitumor activity of anti-RANKL / PD-1 BsAb requires CD8 + T cells, host PD-1 and IFNc. Compared with the combination of two separate mAbs, the simultaneous targeting of RANKL and PD-1 in the tumor microenvironment (TME) can increase the antitumor efficacy. Also, multiple types of dual immunomodulators have been tested in preclinical or phase I clinical studies. M7824 is bispecific immunotherapy designed to block both PD-L1 and TGF and is undergoing phase I clinical research on the compound. MGD013 is a dual immunomodulator targeting PD-1 and LAG-3. Both targets are expressed on exhausted $\mathrm{T}$ cells and tumorinfiltrating lymphocytes, and inhibition of these two targets has been shown to have a synergistic effect on tumor immunity in mice. And a phase I study of MGD013 started from 2017 and will be finished in August 2020, tested this BsAb among the patients with unrespectable or metastatic neoplasm [49,50]. XMBA20717, a BsAb targeting PD-1and CTLA-4, also been tested in preclinical studies and the results suggested that dual blockade of both PD-1and CTLA-4 with XMBA20717 significantly enhanced $t$ cell proliferation and activation and antitumor activity in vivo [49].

\section{PD1/PD-L1 mAb with vaccine}

Cancer vaccine has four major types, including peptide vaccine, vector-based vaccine, whole-cell vaccine and dendritic cell vaccine. All the cancer vaccines were designed to deliver antigenic epitopes or tumor specific antigen to the patients and induce the tumor-specific effector $\mathrm{T}$ cells and generate an antitumor response. Vaccines may be an effective way to produce effector $\mathrm{T}$ cells and induce their infiltration into tumors. However, whether the immune responses induced by vaccines play a role in the tumor microenvironment is still controversial. In some case, patients won't respond to the immunotherapeutic mAbs as monotherapy because of the tumor reduced the expression of checkpoint target for immunotherapy. Pancreatic cancer that shows high tolerance can generate an "immune quiescent" tumor 
microenvironment. The expression of PD-L1 is deficient, and pancreatic carcinoma cells do not respond to PD-1 or PD-L1 antibody mono treatment. After vaccine treatment, vaccine-induced effector $\mathrm{T}$ cells infiltrated into the pancreatic ductal adenocarcinoma (PDA), the inhibition from PD-1 and PD-L1 was disrupted by checkpoint inhibitor treatment. By targeting PD-L1/PDL1 signalling to PDA tumor cells and monocytes (Mo) induced by vaccine-based therapies, anti-PD-1/PD-L1 therapeutic antibodies can enhance vaccine-induced antitumor immune responses [51].

Conceivably, according to all the previous findings, a combination of vaccine-based therapy with anti-PD1/PD-L1 mAb therapy may synergistically induce an antitumor immune response. A previous study on breast cancer combined anti-PD-1 antibody with a multipeptide vaccine and treated the breast tumor bearing mice by this combination strategy, and the result showed that the survival time had been expended [52]. Another research on colon cancer and pancreatic cancer with mice model combined PD-L1/PD-L1 antibodies with GM-CSF secreting cancer vaccine (GVAX) together. On both models, the results showed that checkpoint blocker treatment enhances the activities of GVAX [53].

In clinical trials, two phase 1 studies about melanoma treatment have been performed. One was aimed to test the safety of combination therapy that combined nivolumab with a multi-peptide vaccine to treat patients have metastatic melanoma with HMB-45, NY-ESO-1, and/or MART-1 positive resected tumor. The results demonstrated that it's a safe combination that can be used to treat melanoma [54]. Another trial also tried to test the safety and efficacy of combine nivolumab with the vaccine to treat the ipilimumab-refractory or -naive melanoma. Most Patients get a good result after got the combination therapy, some can respond to ipilimumab after the treatment $[35,54,55]$.

\section{Discussion}

Combined treatment of immune checkpoints is widely used. Although these immune checkpoint inhibitors have improved the effectiveness of patient treatment, however, preliminary clinical trial data show that the immune checkpoint treatment has not reached the expected positive response rate, and many of the previous hypotheses have also been clinically questioned. Nonetheless, in controlling the disease progression of certain advanced cancers, a large number of immune checkpoint antibody combination treatments have indeed achieved good results. As the primary and acquired tolerance mechanisms of immune checkpoint inhibitors are studied in depth, the combined treatment regimen will be used in more clinical trials. In addition to the combined treatment of CTLA-4 and PD-1 antibodies, the combined treatment also includes immune interference factors in the tumor microenvironment such as toll-like receptor antagonists, tumor lytic viruses, antibodies that block $\mathrm{T}$ cell rejection, and suppressive immunity Cells such as Tregs or macrophages. Combined treatment of these immune factors on the one hand enhances the ability of T cells to clear tumor cells, on the other hand, it also increases the number of $\mathrm{T}$ cells around the tumor tissue, thereby cooperating with immune checkpoint antibodies to inhibit tumor progression.

In addition, other treatment methods such as radiotherapy, chemotherapy, tumor-targeted therapy, cancer vaccines and other joint applications have also been shown to modulate the activity of tumor microenvironmental immune factors and have a synergistic effect with immune checkpoints to achieve enhanced antitumor efficacy the role. However, there is a lack of systematic research on the mechanism of combination therapy, and further elaboration of its inherent molecular mechanism is essential to improve the effectiveness of tumor immunotherapy. Improving the efficiency of tumor therapy with PD-1 inhibitors and reducing the development of their tolerance are urgent problems that need to be solved in current cancer immunotherapy. Many clinical researchers and basic investigators have stated that the combination of PD-1 inhibitors and other antitumor drugs is significantly better than monotherapy and has proved that the resistance of PD-1 inhibitors can be increased by regulating the metabolism of $\mathrm{T}$ cells. It is believed that with the development of new therapy method and immune-biotechnology, combination therapy of antiPD1/PD-L1 mAbs will has better effectiveness on cancer treatment and has a wider development space.

\section{References}

1. S. M. Ansell et al., N. Engl. J. Med. 372, pp. 311319, (2015)

2. M. E. Keir, M. J. Butte, G. J. Freeman, and A. H. Sharpe, Annu. Rev. Immunol. 26, pp. 677-704, (2008)

3. M. Zhang, K. Liu, and M. Wang, RSC Adv. 9, 33903-33911, (2019)

4. J. Yang and L. Hu, Res. Rev. 39, 265-301 ( 2019)

5. W. Zou, J. D. Wolchok, and L. Chen, Sci. Transl. Med. 8, 328rv4-328rv4 (2016)

6. J. Tang, A. Shalabi, and V. M. Hubbard-Lucey, Ann. Oncol. Off. J. Eur. Soc. Med. Oncol. 29, 84-91, (2018)

7. J. Xin Yu, J. P. Hodge, C. Oliva, S. T. Neftelinov, V. M. Hubbard-Lucey, and J. Tang, Nat. Rev. Drug Discov., 19, 163-164 (2020)

8. C. Wang, P. Kulkarni, and R. Salgia, Mol. Ther. Oncolytics, 13, 1-6 (2019)

9. S. Joshi and D. L. Durden, J. Oncol. 2019, 1-18 (2019)

10. H. Wei, Z. Zhao, Y. Wang, J. Zou, Q. Lin, and Y. Duan, ACS Appl. Mater. Interfaces, 11, 4647946489 (2019)

11. G. Kroemer, L. Galluzzi, O. Kepp, and L. Zitvogel, Annu. Rev. Immunol. 31, 51-72 (2013)

12. R. Ramakrishnan et al. J. Clin. Invest. 120, 11111124 (2010) 
13. S. V. Liu et al., Eur. J. Cancer, 101, 114-122 (2018)

14. N. A. Rizvi et al., J. Clin. Oncol., 34, 2969-2979 (2016)

15. E. Suzuki, Clin. Cancer Res., 11, 6713-6721 (2005)

16. W. M. Liu, D. W. Fowler, P. Smith, and A. G. Dalgleish, Br. J. Cancer, 102, 115-123 (2010)

17. C. Lazzari et al., Ther. Adv. Med. Oncol., 10, 175883591876209 (2018)

18. T. Kordbacheh, J. Honeychurch, F. Blackhall, C. Faivre-Finn, and T. Illidge, Ann. Oncol. 29, 301310 (2018)

19. A. Oweida et al., OncoImmunology, 6, e1356153 (2017)

20. K.-J. Kim, J.-H. Kim, S. J. Lee, E.-J. Lee, E.-C. Shin, and J. Seong, Oncotarget, 8, 41242-41255, (2017)

21. T. Aguilera, M. Rafat, M. Kariolis, R. von Eyben, E. Graves, and A. Giaccia, J. Immunother. Cancer, 3, P345, 2051-1426-3-S2-P345 (2015)

22. A. B. Sharabi et al., Cancer Immunol. Res. 3, 345355 (2015)

23. L. Deng et al., J. Clin. Invest. 124, 687-695, (2014)

24. K. A. Ahmed et al., Int. J. Radiat. Oncol. 93, S57, (2015)

25. C. A. Chambers, M. S. Kuhns, J. G. Egen, and J. P. Allison, Annu. Rev. Immunol., 19, 565-594 (2001)

26. R. V. Parry et al., Mol. Cell. Biol. 25, 9543-9553, (2005)

27. M. F. Krummel and J. P. Allison, J. Exp. Med. 182, 459-465 (1995)

28. F. S. Hodi et al., N. Engl. J. Med. 363, 711-723 (2010)

29. C. Robert et al., N. Engl. J. Med. 372, 2521-2532 (2015)

30. C. A. Chambers, T. J. Sullivan, and J. P. Allison, Immunity, 7, 885-895 (1997)

31. H. Nishimura, Science, 291, 319-322 (2001)

32. H. Nishimura and T. Honjo, Trends Immunol. 22, 265-268 (2001)

33. F. S. Hodi et al., Lancet Oncol., 17, 1558-1568 (2016)

34. J. D. Wolchok et al., N. Engl. J. Med., 377, 13451356 (2017)

35. F. S. Hodi et al., Lancet Oncol. 19, 1480-1492 (2018)

36. H. J. Hammers et al., J. Clin. Oncol. 35, 3851-3858 (2017)

37. D. Cella et al., Lancet Oncol., 20, 297-310 (2019)

38. C. Wang, G. H. Y. Lin, A. J. McPherson, and T. H. Watts, Immunol. Rev. 229, 192-215 (2009)

39. J. Bukczynski, T. Wen, and T. H. Watts, Eur. J. Immunol. 33, 446-454 (2003)

40. K. Saoulli et al., J. Exp. Med. 187, 1849-1862 (1998)

41. N. H. Segal et al., Clin. Cancer Res. 24, 1816-1823 (2018)
42. S. Chen et al., Cancer Immunol. Res. 3, 149-160 (2015)

43. P. Romero, J. Immunother. Cancer 4, 7 (2016)

44. D. S. Shin et al., Cancer Discov. 7, 188-201 (2017)

45. D. Mittal, D. Vijayan, and M. J. Smyth, Cancer Discov. 8, 1066-1068 (2018)

46. I. S. Nijhof et al., Blood, 128, 959-970 (2016)

47. D. M. Anderson et al., Nature 390, 175-179 (1997)

48. D. L. Lacey et al., Nat. Rev. Drug Discov., 11, 401419 (2012)

49. E. Dahlén, N. Veitonmäki, and P. Norlén, Ther. Adv. Vaccines Immunother. 6, 3-17 (2018)

50. S.-R. Woo et al., Cancer Res. 72, 917-927 (2012)

51. J. Kleponis, R. Skelton, and L. Zheng, 12, p. 8 (2015)

52. L. Karyampudi et al., Cancer Res. 74, 2974-2985 (2014)

53. B. Li, M. VanRoey, C. Wang, T. T. Chen, A. Korman, and K. Jooss, Clin. Cancer Res., 15, 1623 1634 (2009)

54. G. T. Gibney et al., Clin. Cancer Res. 21, 712-720 (2015)

55. J. D. Wolchok et al., N. Engl. J. Med. 369, 122-133, (2013) 